\title{
Dissecting clinical heterogeneity of bipolar disorder using multiple polygenic risk scores
}

Brandon J. Coombes (1) ', Matej Markota², J. John Mann ${ }^{3,4}$, Colin Colby ${ }^{1}$, Eli Stahl $\mathbb{C}^{5,6}$, Ardesheer Talati, ${ }^{3,7}$, Jyotishman Pathak', Myrna M. Weissman ${ }^{3,7,9}$, Susan L. McElroy ${ }^{10}$, Mark A. Frye ${ }^{2}$ and Joanna M. Biernacka (D) ${ }^{1,2}$

\begin{abstract}
Bipolar disorder (BD) has high clinical heterogeneity, frequent psychiatric comorbidities, and elevated suicide risk. To determine genetic differences between common clinical sub-phenotypes of $\mathrm{BD}$, we performed a systematic polygenic risk score (PRS) analysis using multiple PRSs from a range of psychiatric, personality, and lifestyle traits to dissect differences in BD sub-phenotypes in two BD cohorts: the Mayo Clinic BD Biobank $(N=968)$ and Genetic Association Information Network $(N=1001)$. Participants were assessed for history of psychosis, early-onset $B D$, rapid cycling (defined as four or more episodes in a year), and suicide attempts using questionnaires and the Structured Clinical Interview for DSM-IV. In a combined sample of 1969 bipolar cases (45.5\% male), those with psychosis had higher PRS for SCZ (OR = 1.3 per S.D.; $p=3 \mathrm{e}-5)$ but lower PRSs for anhedonia $(\mathrm{OR}=0.87 ; p=0.003)$ and $\mathrm{BMI}(\mathrm{OR}=0.87$; $p=0.003)$. Rapid cycling cases had higher PRS for ADHD (OR $=1.23 ; p=7 \mathrm{e}-5)$ and $\mathrm{MDD}(\mathrm{OR}=1.23 ; p=4 \mathrm{e}-5)$ and lower BD PRS $(\mathrm{OR}=0.8 ; p=0.004)$. Cases with a suicide attempt had higher PRS for MDD $(\mathrm{OR}=1.26 ; p=1 \mathrm{e}-6)$ and anhedonia $(\mathrm{OR}=1.22 ; p=2 \mathrm{e}-5)$ as well as lower PRS for educational attainment $(\mathrm{OR}=0.87 ; p=0.003)$. The observed novel PRS associations with sub-phenotypes align with clinical observations such as rapid cycling BD patients having a greater lifetime prevalence of ADHD. Our findings confirm that genetic heterogeneity contributes to clinical heterogeneity of $\mathrm{BD}$ and consideration of genetic contribution to psychopathologic components of psychiatric disorders may improve genetic prediction of complex psychiatric disorders.
\end{abstract}

\section{Introduction}

Many psychiatric disorders have moderate to high heritability; however, the genetics of psychiatric disorders are complex and highly polygenic, with each risk variant only conferring a small effect ${ }^{1}$. Psychiatric disorders also have a high level of overlapping clinical heterogeneity, with shared genetic risk explaining some of the clinical overlap, and certain combinations of alleles may contribute to the same psychopathological symptoms in multiple psychiatric disorders. Furthermore, some psychiatric disorders may lie on a continuum rather than being disorders with distinct genetics and biological mechanisms ${ }^{2,3}$.

Correspondence: Brandon J. Coombes (coombes.brandon@mayo.edu) ${ }^{1}$ Department of Health Sciences Research, Mayo Clinic, Rochester, MN, USA 2Department of Psychiatry and Psychology, Mayo Clinic, Rochester, MN, USA Full list of author information is available at the end of the article
To accommodate this genetic complexity, investigations of psychiatric disorders have increasingly relied on polygenic risk scores (PRSs), leveraging knowledge from prior large genome-wide association studies (GWASs) to predict genetic risk of particular disorders in a new sample ${ }^{4}$. When the PRS for one disorder is predictive of a second disorder, this indicates a common polygenic contribution to the two disorders ${ }^{5}$.

Bipolar disorder (BD) is a complex illness with heterogeneous clinical presentation, and apparent subphenotypes often have a different course of illness, prognosis, and treatment response ${ }^{6-9}$. In order to personalize treatment, it is crucial to better understand biological underpinnings of $\mathrm{BD}$ clinical sub-phenotypes. One approach is to examine potential relationships of clinical phenotypes to different genetic profiles.

\section{(c) The Author(s) 2020}

(c) (i) Open Access This article is licensed under a Creative Commons Attribution 4.0 International License, which permits use, sharing, adaptation, distribution and reproduction cc) in any medium or format, as long as you give appropriate credit to the original author(s) and the source, provide a link to the Creative Commons license, and indicate if changes were made. The images or other third party material in this article are included in the article's Creative Commons license, unless indicated otherwise in a credit line to the material. If material is not included in the article's Creative Commons license and your intended use is not permitted by statutory regulation or exceeds the permitted use, you will need to obtain permission directly from the copyright holder. To view a copy of this license, visit http://creativecommons.org/licenses/by/4.0/. 
Historically, the relationship between schizophrenia (SCZ) and BD has shaped classification systems in psychiatry $^{10}$. The corresponding link between phenotype and genetics was recently established with the demonstration that BD patients with a history of psychosis, particularly mood incongruent psychosis and psychosis during mania, have increased genetic risk for $\mathrm{SCZ}^{11-16}$. However, it is well recognized that $\mathrm{BD}$ genetically overlaps-and has high clinical comorbidity with-other major psychiatric conditions, including major depressive disorder (MDD), attention deficit and hyperactivity disorder (ADHD), anxiety disorders, post-traumatic stress disorder (PTSD), obsessive compulsive disorder (OCD), borderline personality disorder, and substance use disorders ${ }^{17-24}$. While significant advances have been made in understanding the genetic relationship between $\mathrm{BD}$ psychotic subphenotypes and $\mathrm{SCZ}^{11-16}$, little is known about how genetic risks for other psychiatric disorders as well as important personality and lifestyle traits such as body mass index (BMI), risk-taking, and neuroticism relates to psychosis or other BD clinical sub-phenotypes.

The goal of this study was to systematically test if PRSs for major psychiatric conditions and other traits related to $\mathrm{BD}$ are predictors of distinct $\mathrm{BD}$ sub-phenotypes, in particular with regards to psychosis, age-of-onset, rapid cycling, and suicidal behavior. Understanding the shared genetic risk factors between BD clinical sub-phenotypes and other comorbid conditions may contribute to psychiatric clinical classification systems with a more biologically informed nosological system ${ }^{25}$.

\section{Methods and materials}

\section{Studies}

\section{Mayo Clinic Bipolar Disorder Biobank}

The Mayo Clinic Bipolar Disorder Biobank collection has been described in previous papers ${ }^{9,11,26}$. We restricted our analyses to cases with European ancestry $(N=968)$, because PRSs derived from GWASs of participants with European ancestry perform much worse in non-European ancestries ${ }^{27}$. Sub-phenotypes were determined using the Structured Clinical Interview for DSM-IV (SCID) ${ }^{28}$ as well as a patient questionnaire (Supplementary Table 1). These were conducted by research coordinators that were trained and certified on using the tools. Any discrepancy between SCID diagnoses and diagnoses in medical records was reviewed by a licensed psychiatrist.

\section{Genetic Association Information Network}

The Bipolar Disorder Genome Study Consortium conducted a GWAS of BD as part of the GAIN ${ }^{29}$. We obtained the data from dbGaP (phs000017.v3.p1), and restricted our analyses to cases with European ancestry $(N=1001)$. All cases met criteria for DSM-IV-defined bipolar I disorder (BD-I). Subjects recruited at different times were interviewed with a one-time Diagnostic Interview for Genetic Studies 2, 3, or 4 (DIGS 2, 3, 4) conducted by study coordinators (Supplementary Table 1).

\section{Genotyping and quality control Mayo Clinic Bipolar Biobank}

Genotyping and genetic data quality control of this sample was previously described as part of a larger casecontrol study ${ }^{11}$. Briefly, the Illumina ${ }^{\circledR}$ HumanOmniExpress platform (Illumina ${ }^{\circledR}$, San Diego, CA, USA) was used to genotype $1046 \mathrm{BD}$ cases. For quality control purposes, we excluded subjects with $<98 \%$ call rate and related subjects. Single-nucleotide polymorphisms (SNPs) with call rate $<98 \%, \mathrm{MAF}<0.01$, and those not in Hardy-Weinberg Equilibrium (HWE; $P<1 \mathrm{e}-06$ ) were removed. After these steps 643011 SNPs and 968 subjects remained.

\section{GAIN}

Genotyping and quality control procedures for the GAIN-BD data were previously described by Smith et al. $^{30}$, Briefly, the Affymetrix ${ }^{\circledR}$ Genome-Wide Human SNP Array 6.0 platform (ThermoFischer Scientific, Waltham, MA, USA) was used to genotype cases and after excluding SNPs with call rate $<98 \%, \mathrm{MAF}<0.01$, and those not in HWE, 726,315 SNPs and 1001 subjects of European ancestry remained.

\section{Imputation}

Genotypes in both the GAIN and Mayo Clinic samples were imputed to the 1000 genomes reference panel, as previously described for the GAIN sample9. Specifically, SHAPEIT $^{31}$ was used for haplotype phasing and imputation was performed using IMPUTE2.2.2 ${ }^{32}$ with the 1000 genome project reference data (phase 1 data, all populations). Dosage data was converted to best guess genotype for the well-imputed (dosage $R^{2}>0.8$ ) and common (MAF > 0.01) SNPs, resulting in more than 5 million SNPs in both datasets.

\section{Polygenic risk scores}

PRSs were included in the analysis if: (1) there was evidence of significant genetic correlation of the trait with $\mathrm{BD}$ and (2) we had at least $80 \%$ power to detect PRS association in a general case-only analysis of our data assuming $50 \%$ prevalence of the sub-phenotype. We began by considering PRSs for major psychiatric disorders $\left(\mathrm{BD}^{33}, \mathrm{SCZ}^{34}, \mathrm{MDD}^{35}, \mathrm{ADHD}^{36}\right.$, anxiety ${ }^{37}, \mathrm{PTSD}^{19}$, $\mathrm{OCD}^{38}$, anorexia nervosa ${ }^{39}$, alcohol use disorder ${ }^{40}$, and insomnia ${ }^{41}$ ) and personality and lifestyle traits related to BD (alcohol consumption ${ }^{40}$, educational attainment $(\mathrm{EA})^{42}$, risk-taking ${ }^{43}$, subjective well-being ${ }^{44}$, neuroti$\operatorname{cism}^{45}$, anhedonia ${ }^{46}$, and body mass index $\left.(\mathrm{BMI})^{47}\right)$. The 
GWAS summary statistics were restricted to wellimputed variants $(\mathrm{INFO}>0.9)$ when information on imputation quality was available.

Using linkage disequilibrium (LD) score regression ${ }^{48}$, we estimated the genetic correlation of the above traits with $\mathrm{BD}^{33}$ (Supplementary Table 2). Insomnia and alcohol consumption did not have significant genetic correlation with $\mathrm{BD}$ and were therefore excluded from further analysis.

Using the R package AVENGEME ${ }^{49}$, we estimated that training sample sizes of 20,000 would achieve at least $80 \%$ power in our analysis assuming moderate overlap of the trait with the sub-phenotype (genetic covariance $=0.1$ ), high polygenicity (\# of independent SNPs $=20,000$ ), and $0.005 \alpha$-level to account for multiple testing. The study of OCD included an effective sample size of $<4000$ and was thus excluded from further analysis. The final list of PRSs that were tested for association with BD sub-phenotypes is shown in Supplementary Table 2.

For traits that satisfied our inclusion criteria, the PRScontinuous shrinkage $(\mathrm{CS})^{50}$ auto setting was applied to estimate SNP weights using a fully Bayesian shrinkage approach that shrinks SNP effects with a continuous shrinkage prior. This setting allows the algorithm to learn the global shrinkage parameter from the data to create one set of weights per PRS and therefore does not require a validation dataset. This setting also reduces the multiple testing of standard PRS analyses that search over many $p$-value thresholds ${ }^{51}$. PLINK version $1.9^{52}$ was used to create PRSs using the shrunken SNP weights. The PRSs were then standardized to have a mean of zero and standard deviation (SD) of one.

\section{Statistical analyses}

In each dataset, we performed principal components (PCs) analysis of the genotyped SNPs and kept the first four PCs to be used as within-study nested covariates in subsequent PRS association analyses. In all models, study indicator and an interaction of study and within-study PCs were included as covariates to control for population stratification. All 12 PRSs were individually modeled using a multivariate logistic regression model with each subphenotype (psychosis, early-onset $\mathrm{BD}$, rapid cycling, and attempted suicide) as the outcome.

We used 10,000 permutations to find the significance threshold to control the false positive rate testing for association with each sub-phenotype with 14 PRSs $(\alpha=0.005)$ as well as the family-wise error rate $(\alpha=0.001)$. For each sub-phenotype, we also included all significant PRSs $(p<0.005)$ in a joint model, to estimate the relative contribution of the PRSs after adjusting for other important PRSs. We report the variance explained in the sub-phenotype by each PRS after adjustment for other PRSs using Nagelkerke's pseudo- $R^{2}$ statistic. All statistical analyses were performed in R 3.5.2.

\section{Results}

\section{Sample description}

Table 1 summarizes the demographic and subphenotype information of each study. There was a difference in the sex distribution between the two samples. The GAIN study only included BD type I cases, and the distributions were also significantly different for all subphenotypes besides attempted suicide. The GAIN-BD cases had a higher rate of psychosis and early-onset BD, while Mayo Clinic cases had higher rates of rapid cycling, which is more prevalent in women ${ }^{53}$.

Figure 1 shows a forest plot of the significant PRS associations with each sub-phenotype further broken down by study. Further detailed results for each subphenotype can be found in Supplementary Tables 3-6.

\section{Psychosis}

Cases with psychosis versus no psychosis had higher PRSs for SCZ $(\mathrm{OR}=1.3,95 \%$ CI 1.15-1.48; $p$-value $=$ 3.5e-5), but lower PRSs for anhedonia (OR $=0.87,95 \% \mathrm{CI}$ $0.79-0.95 ; p$-value $=0.003)$, and $\mathrm{BMI}(\mathrm{OR}=0.87,95 \% \mathrm{CI}$ $0.79-0.95 ; p$-value $=0.004)$. These three PRSs explained $2.6 \%$ of the variation in psychosis in the joint model. While anhedonia is a component of MDD and the two PRSs are positively correlated $(r=0.41)$, the PRS for MDD was not associated with psychosis in BD $(\mathrm{OR}=$ 0.96, 95\% CI 0.87-1.06; $p$-value $=0.45$ ).

\section{Early-onset BD}

Higher PRSs for risk-taking $(\mathrm{OR}=1.21,95 \% \mathrm{CI}$ $1.09-1.35 ; p$-value $=0.0005 ;$ adj. Nagelkerke's $\left.R^{2}=0.8 \%\right)$ and anhedonia $(\mathrm{OR}=1.16,95 \%$ CI 1.05-1.29; $p$-value $=$ 0.0047 ; adj. Nagelkerke's $R^{2}=0.8 \%$ ) were observed in cases with early-onset BD compared to cases that developed BD after age 18.

\section{Rapid cycling}

Cases with rapid cycling versus those without rapid cycling had higher ADHD PRS (OR $=1.23,95 \%$ CI $1.11-1.36 ; p$-value $=7 \mathrm{e}-5 ;$ adj. Nagelkerke's $\left.R^{2}=0.8 \%\right)$, MDD PRS (OR $=1.23$, 95\% CI 1.11-1.36; $p$-value $=4 \mathrm{e}-5$; adj. Nagelkerke's $R^{2}=0.5 \%$ ), PTSD PRS (OR $=1.28,95 \%$ CI $1.14-1.44 ; p$-value $=4 \mathrm{e}-5 ;$ adj. Nagelkerke's $R^{2}=$ $0.7 \%)$, and PRS for anxiety (OR $=1.19,95 \%$ CI $1.07-1.33$; $p$-value $=0.001$; adj. Nagelkerke's $\left.R^{2}=0.1 \%\right)$. Cases with rapid cycling also had lower BD PRSs $(\mathrm{OR}=0.80,95 \% \mathrm{CI}$ $0.68-0.93 ; p$-value $=0.004$; adj. Nagelkerke's $R^{2}=0.9 \%$ ). The five PRSs explained $3.9 \%$ of the variation in rapid cycling when included in one model.

\section{Attempted suicide}

The genetic risk for MDD $(\mathrm{OR}=1.26,95 \% \mathrm{CI}$ $1.15-1.39 ; p$-value $=1 \mathrm{e}-6 ;$ adj. Nagelkerke's $\left.R^{2}=0.7 \%\right)$ and anhedonia $(\mathrm{OR}=1.22,95 \% \mathrm{CI} 1.12-1.34 ; p$-value $=$ 
Table 1 Table of sub-phenotypes and sex for each study.

\begin{tabular}{|c|c|c|c|c|c|}
\hline Variable & Value & $\begin{array}{l}\text { All } \\
N=1969(\%)\end{array}$ & $\begin{array}{l}\text { GAIN } \\
N=1001(\%)\end{array}$ & $\begin{array}{l}\text { Mayo } \\
N=968(\%)\end{array}$ & $p$-value \\
\hline \multirow[t]{2}{*}{ Sex } & Male & 895 (45.5) & $500(50.0)$ & 395 (40.8) & 0.003 \\
\hline & Female & $1074(54.5)$ & $501(50.0)$ & $573(59.2)$ & \\
\hline \multirow[t]{2}{*}{ BD type } & BD-II & $263(13.4)$ & - & $263(27.2)$ & - \\
\hline & BD-I & 1706 (86.6) & 1001 & 705 (72.8) & \\
\hline \multirow[t]{3}{*}{ Psychosis } & No Psychosis & $880(47.3)$ & $344(34.9)$ & $536(61.3)$ & $3 e-7$ \\
\hline & Psychosis & $980(52.7)$ & $642(65.1)$ & $338(38.7)$ & \\
\hline & Missing & 109 & 15 & 94 & \\
\hline \multirow[t]{3}{*}{ Age-of-onset } & $>18 \mathrm{yrs}$ & $1292(70.0)$ & $569(60.3)$ & $723(80.0)$ & $1 e-13$ \\
\hline & $<19 \mathrm{yrs}$ & $555(30.1)$ & $374(39.7)$ & $181(20.0)$ & \\
\hline & Missing & 122 & 58 & 64 & \\
\hline \multirow[t]{3}{*}{ Rapid Cycling } & No & $903(50.1)$ & $508(60.3)$ & $395(41.1)$ & $3 e-11$ \\
\hline & Yes & 901 (49.9) & 334 (39.7) & 567 (58.9) & \\
\hline & Missing & 165 & 159 & 6 & \\
\hline Suicide & None & $1115(57.2)$ & $554(56.5)$ & $561(58.0)$ & 0.619 \\
\hline \multirow[t]{2}{*}{ attempts } & $1+$ & $833(42.8)$ & $427(43.5)$ & $406(42.0)$ & \\
\hline & Missing & 21 & 20 & 1 & \\
\hline
\end{tabular}

$P$-value is for a $X^{2}$ test of differences between GAIN and Mayo Clinic BD-I subject sub-phenotypes.

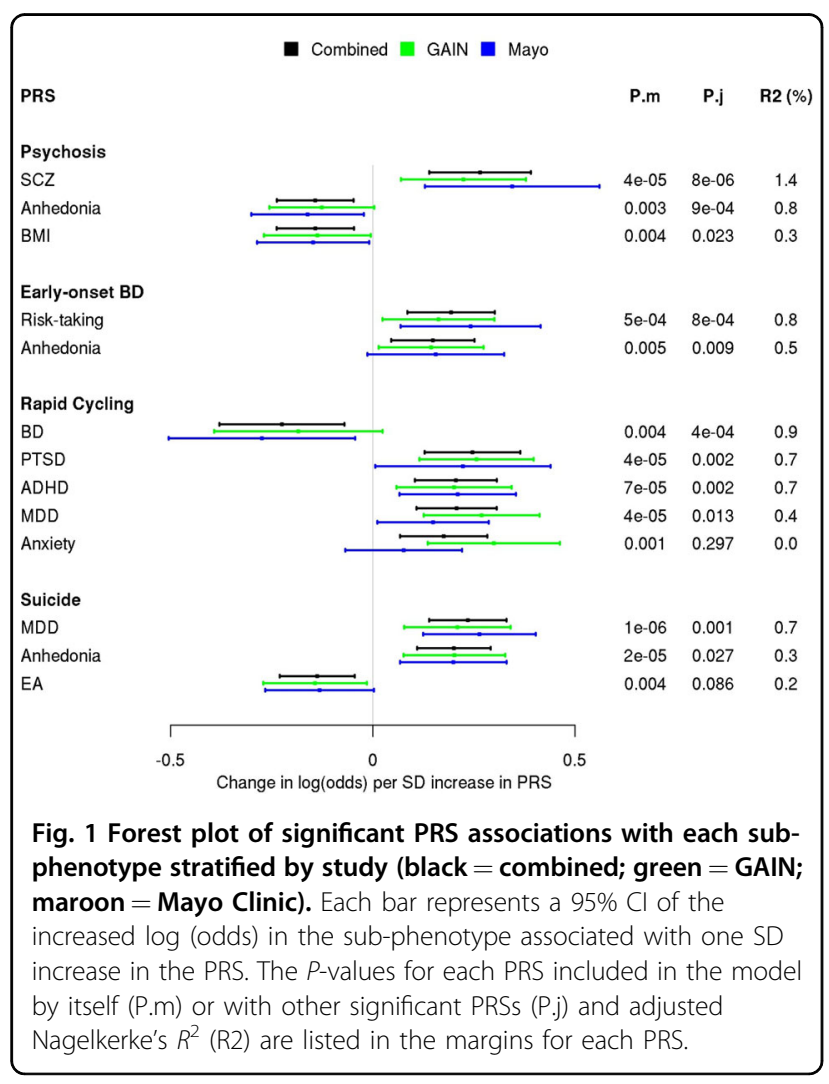

2e-5; adj. Nagelkerke's $R^{2}=0.3 \%$ ) was higher in cases with at least one suicide attempt versus those with none. Cases with an attempted suicide also had a lower PRS for EA $(\mathrm{OR}=0.87,95 \%$ CI $0.79-0.96 ; p$-value $=0.0036 ;$ adj. Nagelkerke's $\left.R^{2}=0.2 \%\right)$. The three PRSs explained a total of $2.3 \%$ of the variation when included in one model, but only the MDD PRS remained significant after accounting for the other PRS associations.

\section{Discussion}

To our knowledge, this is the first PRS dissection of clinical sub-phenotypes in BD that is comprehensive with respect to the range of psychiatric, personality, and lifestyle phenotypes for which genetic liabilities were estimated and used to predict the BD sub-phenotypes. Previous analyses of BD sub-phenotypes of psychosis, early-onset $\mathrm{BD}$, and suicide focused on genetic liability to the major psychiatric diagnoses of $\mathrm{BD}, \mathrm{SCZ}$, and $\mathrm{MDD}^{6,11,12,16,54}$. Here, we took an expanded agnostic approach to PRS analysis by using many different PRSs beyond just these three to more systematically test for PRS association with clinically important sub-phenotypes of $\mathrm{BD}$, including rapid cycling. Importantly, the contribution of each PRS to a sub-phenotype was assessed after adjusting for the other PRSs' contributions, thereby assessing how predictive a genetic risk is above and 
beyond other correlated genetic risks (Supplementary Fig. $1)$. Our results were highly comparable in the two cohorts, lending greater confidence to the conclusions (Fig. 1). Overall, we find that the different BD clinical subphenotypes have different profiles of PRS associations with major psychiatric conditions.

\section{BD with psychosis}

Previously, using the Mayo Clinic sample, we showed that $\mathrm{BD}$ patients with a history of psychosis during mania had higher genetic risk for $\mathrm{SCZ}^{11}$. Here, this finding is replicated in the GAIN cohort. This finding was also reported by Ruderfer et al. ${ }^{16}$, in a larger study that included both the Mayo Clinic and GAIN-BD cohorts. However, in addition to this relationship, in the present study we also found that BD cases that have not experienced psychotic symptoms had higher genetic scores for anhedonia and BMI. Association of higher genetic risk for anhedonia with a subtype of BD without psychotic features implies that a patient with more genetic predisposition for anhedonia during major depressive episodes is less likely to include episodes with psychotic features. In fact, rates of psychotic features are higher in BD compared with MDD, and familial studies show a greater heritability of psychotic features in $\mathrm{BD}$ relative to in other mood disorders ${ }^{55}$. Interestingly, we did not observe a significant association with MDD PRS despite a strong genetic correlation between anhedonia and MDD. This may underscore the importance of relying on core symptoms in these analyses, instead of using more complex and syndromal entities like MDD. The relationship between BMI and psychosis is complex and influenced by heritable, environmental, and iatrogenic factors. Over the course of illness, most patients with BD and psychosis gain weight, which contributes to morbidity and mortality $^{56,57}$. Our finding that BD patients with psychosis have lower genetic predisposition to elevated BMI than BD patients without psychosis suggest that weight gain in those with psychosis may be a side effect of medications, which is in line with historic observations predating discovery of antipsychotic medications ${ }^{58}$. However, the complex relationship between $\mathrm{BD}$ and greater body weight needs to be further explored in the context of subphenotypes and use of atypical antispychotics or lithium. Likewise, future studies should further elaborate on the association between BD psychosis sub-phenotypes, e.g. $\mathrm{BD}$ with mood congruent vs incongruent psychosis, and PRSs investigated here.

\section{Early-onset BD}

We found evidence that higher genetic liability for risktaking behavior was associated with early-onset $\mathrm{BD}$, but no evidence that genetic risk for SCZ or BD were associated with age of onset of illness. A previous study of polygenic associations with age-of-onset of $\mathrm{BD}$ also showed no association of SCZ or BD genetic risk with both a dichotomous sub-phenotype, as defined in our study, or continuous age-of-onset ${ }^{59}$. Risk-taking is a hallmark feature of normative adolescence but is also commonly seen in mania. There are several possible explanations for the risk-taking PRS and early-onset BD association found in this study. Perhaps the simplest explanation is that youth with particularly high propensity for risk-taking behaviors come to clinical attention earlier and subsequently have BD identified at an earlier age. However, there are several potential limitations that may have affected these findings. Due to the way the data were collected, for this study, age of onset was dichotomized based on a cutoff age of 18, which may have reduced power. Also, our early-onset BD definition did not differentiate between age of first manic and first depressive episodes. Furthermore, given the high genetic and clinical overlap between $\mathrm{BD}$ and other conditions investigated here (e.g. ADHD), a study of age-of-onset of any psychiatric disorder/symptom rather than just $\mathrm{BD}$ could be informative. It is of note that earlier onset MDD is associated with more pronounced aggressive/impulsive traits $^{60}$. Nevertheless, the observed association of risktaking PRS with early vs. late onset $\mathrm{BD}$ is intriguing and warrants further investigation.

\section{BD with rapid cycling}

Previous clinical studies have shown a strikingly higher clinical comorbidity rate of ADHD in BD patients with rapid cycling compared to non-rapid cycling $\mathrm{BD}$ patients ${ }^{61}$. While the general genetic association between ADHD and $\mathrm{BD}$ has been described before ${ }^{17}$, our results are the first study to show possible genetic underpinnings for this specific rapid cycling BD and ADHD association. We also found a strong association of MDD genetic risk with rapid cycling. This implies that genetic variation related to ADHD and MDD may also be related to episode frequency in $\mathrm{BD}$, and that comorbid ADHD and more depressive episodes would be clinically associated with the rapid cycling form of $\mathrm{BD}$, though the predominant directionality of mood episodes was not discernible from the available data. Rapid cycling BD has been reported to have more episodes of major depression and a higher rate of parental MDD compared with nonrapid cycling $\mathrm{BD}^{62}$, which is consistent with our PRS association findings. Finally, rapid cycling cases had lower BD PRS as reported in a previous investigation ${ }^{15}$. This could simply reflect that prevalence of rapid cycling in cases ascertained for the sample used in the GWAS of BD by the Psychiatric Genomics Consortium (PGC) was lower than in the two samples included here, but still demonstrates a systematic difference in genetics of rapid cycling and non-rapid cycling BD. 


\section{BD with a history of a suicide attempt}

Our finding of increased MDD genetic load in BD patients with a history of suicide attempts is consistent with a recent study that included both of the Mayo Clinic and GAIN data, which showed that genetic risk factors for MDD increase the risk for suicide trans-diagnostically ${ }^{54}$. BD with a history of suicide attempt having a higher MDD genetic liability is consistent with the clinical observation that suicide attempts are most common during major depressive episodes or mixed states and rare during manic episodes or while euthymic ${ }^{63,64}$. Interestingly, even after adjusting for MDD PRS, we also found that genetic liability for anhedonia is marginally associated with suicide, suggesting that anhedonia may be a particularly relevant factor contributing to suicidality, compared to other components that comprise the MDD syndrome. This is consistent with findings from non-genetic studies, which found that association of anhedonia with suicidality is independent of the association with depression and psychotic features ${ }^{65}$.

\section{Methodological limitations}

The PRSs used in this study are based on data from previously published large scale investigations and are limited by the diagnostic accuracy, recruitment criteria, and methodology of previous studies. The most recent PGC study of $\mathrm{BD}^{33}$ included the cases and controls from the GAIN and Mayo Clinic samples. Sample overlap of testing datasets with training data can create substantial biases in PRS analyses. However, here we studied genetic differences within cases and thus, the sample overlap is not expected to bias our results, because there should be little correlation between case-control status used to build the training models and the within-case sub-phenotypes. However, this is an open research question and requires further methodological study. Thus caution is needed with respect to any interpretation in associations of BD-PRS with sub-phenotypes. Also, because sample sizes in training GWASs vary, the power to detect PRS associations with sub-phenotypes in our analyses was not uniform.

Another limitation is the cross-sectional data collection using SCIDs and patient questionnaires and does not consider developmental trajectories of psychopathology over time. The data also lacks the number, duration, and severity of major depressive and manic episodes to more precisely map the clinical picture onto the PRS profile. Furthermore, sub-phenotypes such as rapid cycling and psychosis could be substance-induced but was not assessed in this study. Finally, it is important to note that no PRS explained a large amount of variation in our analysis. Thus, while the associations identified in this study provide evidence of genetic differences that may underlie clinical subtypes of BD, these PRSs cannot yet be used for purposes of personalized psychiatry.

\section{Conclusion}

Our findings contribute to the understanding of the underlying genetic causes of clinical heterogeneity of $\mathrm{BD}$ and of comorbidity between $\mathrm{BD}$ and other major psychiatric conditions. We find evidence that psychopathologic components of $\mathrm{BD}$, including psychotic symptoms, rapid cycling, and suicidal behavior are linked to the PRSs for related disorders including schizophrenia, ADHD, and MDD, respectively. Finally, larger studies are needed to more precisely map genetic risk factors to clinical subphenotypes. Harmonization of sub-phenotypes across studies is a well-recognized challenge. Nevertheless, such efforts are critical in helping to classify psychiatric disorders more accurately and identify risk of suicide, psychosis, and other adverse outcomes in patients.

\section{Acknowledgements \\ This work was supported by the Marriott Foundation and the Thomas and Elizabeth Grainger Fund in Bipolar Functional Genomics and Drug Development awarded to the Mayo Clinic. Establishment of the Bipolar Disorder Biobank was supported by a generous gift from the Marriot Family and the Mayo Clinic Center for Individualized Medicine. The contributions from authors J.J.M., A.T., and M.M.W. was supported by NIMH ROMH121921 (Wickramaratne and Mann, M.P.I.S).}

\section{Author details \\ ${ }^{1}$ Department of Health Sciences Research, Mayo Clinic, Rochester, MN, USA. ${ }^{2}$ Department of Psychiatry and Psychology, Mayo Clinic, Rochester, MN, USA. ${ }^{3}$ Department of Psychiatry, Columbia University Vagelos College of Physicians \& Surgeons, New York, NY, USA. ${ }^{4}$ Division of Molecular Imaging and Neuropathology, New York State Psychiatric Institute, New York, NY, USA. ${ }^{5}$ Pamela Sklar Division of Psychiatric Genomics, Icahn School of Medicine at Mount Sinai, New York, NY, USA. ${ }^{6}$ Medical and Population Genomics, Broad Institute, Cambridge, MA, USA. ${ }^{7}$ Divisions of Translational Epidemiology, New York State Psychiatric Institute, New York, NY, USA. ${ }^{8}$ Department of Healthcare Policy \& Research, Weill Medical College, Cornell University, New York, NY, USA. ${ }^{9}$ Department of Epidemiology, Mailman School of Public Health, Columbia University, New York, NY, USA. ${ }^{10}$ Lindner Center of HOPE/University of Cincinnati, Cincinnati, $\mathrm{OH}$, USA}

\section{Conflict of interest}

Dr. Frye has received grant support from Assurex Health, Myriad, Pfizer, National Institute of Mental Health (RO1 MH079261), National Institute of Alcohol Abuse and Alcoholism (P20AA017830), and Mayo Foundation; has been a consultant to Janssen Global Services, LLC, Mitsubishi Tanabe Pharma Corporation, Myriad, Sunovion, and Teva Pharmaceuticals; has received CME/ Travel Support/presentation from CME Outfitters Inc. and Sunovian; Mayo Clinic has a financial interest in AssureRx and OneOme. Dr. McElroy is a consultant to or member of the scientific advisory boards of Bracket, MedAvante, Naurex, Shire, and Sunovion. She is a principal or co-investigator on studies sponsored by the Agency for Healthcare Research \& Quality (AHRQ), AstraZeneca, Cephalon, Forest, Marriott Foundation, National Institute of Mental Health, Orexigen Therapeutics, Inc., Shire, and Takeda Pharmaceutical Company Ltd. She is also an inventor on United States Patent No. 6,323,236 B2, Use of Sulfamate Derivatives for Treating Impulse Control Disorders, and along with the patent's assignee, University of Cincinnati, Cincinnati, Ohio, has received payments from Johnson \& Johnson, which has exclusive rights under the patent. In the last 3 years, Dr Weissman has received research funds from $\mathrm{NIMH}$, Templeton Foundation, Brain and Behavior and the Sackler Foundation, and has received royalties for publications of books on interpersonal psychotherapy from Perseus Press, Oxford University Press, on other topics 
from the American Psychiatric Association Press and royalties on the social adjustment scale from Multihealth Systems. None of these represent a conflict of interest.

\section{Publisher's note}

Springer Nature remains neutral with regard to jurisdictional claims in published maps and institutional affiliations.

Supplementary Information accompanies this paper at (https://doi.org/ 10.1038/s41398-020-00996-y).

Received: 17 April 2020 Revised: 10 August 2020 Accepted: 3 September 2020

Published online: 18 September 2020

\section{References}

1. Smoller, J. W. et al. Psychiatric genetics and the structure of psychopathology. Mol. Psychiatry 24, 409-420 (2019).

2. Owen, M. J. \& O'Donovan, M. C. Schizophrenia and the neurodevelopmental continuum:evidence from genomics. World Psychiatry 16, 227-235 (2017).

3. Tesli, M. et al. Polygenic risk score and the psychosis continuum model. Acta Psychiatr. Scand. 130, 311-317 (2014).

4. Martin, A. R., Daly, M. J., Robinson, E. B., Hyman, S. E. \& Neale, B. M. Predicting polygenic risk of psychiatric disorders. Biol. Psychiatry 86, 97-109 (2019).

5. Torkamani, A., Wineinger, N. E. \& Topol, E. J. The personal and clinical utility of polygenic risk scores. Nat. Rev. Genet. 19, 581-590 (2018).

6. Charney A. W. et al. Evidence for genetic heterogeneity between clinical subtypes of bipolar disorder. Transl Psychiatry. 7, e993 (2017).

7. Alda, M. The phenotypic spectra of bipolar disorder. Eur. Neuropsychopharmacol. 14, S94-S99 (2004)

8. Alda, M., Hajek, T., Calkin, C. \& O'Donovan, C. Treatment of bipolar disorder: new perspectives. Ann. Med. 41, 186-196 (2009).

9. Winham, S. J. et al. Bipolar disorder with comorbid binge eating history: a genome-wide association study implicates APOB. J. Affect Disord. 165 151-158 (2014).

10. Kraepelin E. Manic-depressive Insanity And Paranoia: Classics In Psychiatry. (Ayer Company Publishers, Salem (NH), 1921).

11. Markota, M. et al. Association of schizophrenia polygenic risk score with manic and depressive psychosis in bipolar disorder. Transl. Psychiatry $\mathbf{8}$, 188 (2018)

12. Ruderfer, D. M. et al. Polygenic dissection of diagnosis and clinical dimensions of bipolar disorder and schizophrenia. Mol. Psychiatry 19, 1017-1024 (2014).

13. Hamshere, M. L. et al. Polygenic dissection of the bipolar phenotype. Br. J. Psychiatry 198, 284-288 (2011).

14. Allardyce, J. et al. Association between schizophrenia-related polygenic liability and the occurrence and level of mood-incongruent psychotic symptoms in bipolar disorder. JAMA Psychiatry 75, 28-35 (2018).

15. Ruderfer, D. M. et al. Genomic dissection of bipolar disorder and schizophrenia, including 28 subphenotypes. Cell 173, 1705-1715.e16 (2018).

16. Bipolar Disorder and Schizophrenia Working Group of the Psychiatric Genomics Consortium. Genomic dissection of bipolar disorder and schizophrenia, including 28 subphenotypes. Cell 173, 1705-1715.e16 (2018).

17. van Hulzen, K. J. E. et al. Genetic overlap between attention-deficit/hyperactivity disorder and bipolar disorder: evidence from genome-wide association study meta-analysis. Biol. Psychiatry 82, 634-641 (2017).

18. Wozniak, J., Biederman, J., Monuteaux, M. C., Richards, J. \& Faraone, S. V. Parsing the comorbidity between bipolar disorder and anxiety disorders: a familial risk analysis. J. Child Adolesc. Psychopharmacol. 12, 101-111 (2002).

19. Duncan, L. E. et al. Largest GWAS of PTSD $(N=20070)$ yields genetic overlap with schizophrenia and sex differences in heritability. Mol. Psychiatry 23, 666-673 (2018).

20. Yilmaz Z. et al. Examination of the shared genetic basis of anorexia nervosa and obsessive-compulsive disorder. Mol. Psychiatry. https://doi.org/10.1038/ s41380-018-0115-4 (2018).

21. Witt, S. H. et al. Genome-wide association study of borderline personality disorder reveals genetic overlap with bipolar disorder, major depression and schizophrenia. Transl. Psychiatry 7, e1155 (2017).
22. Schulze, T. G. et al. Molecular genetic overlap in bipolar disorder, schizophrenia, and major depressive disorder. World J. Biol. Psychiatry 15, 200-208 (2014).

23. Chen Q. et al. Common psychiatric and metabolic comorbidity of adult attention-deficit/hyperactivity disorder: a population-based cross-sectional study. PLOS ONE 13, e0204516 (2018).

24. McKowen, J. W., Frye, M. A., Altshuler, L. L. \& Gitlin, M. J. Patterns of alcohol consumption in bipolar patients comorbid for alcohol abuse or dependence. Bipolar Disord. 7, 377-381 (2005).

25. Salagre E. et al. Toward precision psychiatry in bipolar disorder: staging 2.0. Front. Psychiatry 9, 641 (2018).

26. Frye, M. A. et al. Development of a bipolar disorder biobank: differential phenotyping for subsequent biomarker analyses. Int. J. Bipolar Disord. 3, 14 (2015).

27. Martin, A. R. et al. Clinical use of current polygenic risk scores may exacerbate health disparities. Nat. Genet. 51, 584-591 (2019).

28. The American Psychiatric Association. Diagnostic And Statistical Manual of Mental Disorders (DSM-5 ${ }^{\circledR}$ ). (American Psychiatric Pub, 2013).

29. GAIN Collaborative Research Group. New models of collaboration in genomewide association studies: the Genetic Association Information Network. Nat Genet. 39, 1045-1051 (2007).

30. Smith, E. N. et al. Genome-wide association study of bipolar disorder in European American and African American individuals. Mol. Psychiatry 14, 755-763 (2009).

31. Delaneau, O., Marchini, J. \& Zagury, J.-F. A linear complexity phasing method for thousands of genomes. Nat. Methods 9, 179-181 (2011).

32. Howie, B., Fuchsberger, C., Stephens, M., Marchini, J. \& Abecasis, G. R. Fast and accurate genotype imputation in genome-wide association studies through pre-phasing. Nat. Genet. 44, 955 (2012).

33. Stahl, E. A. et al. Genome-wide association study identifies 30 loci associated with bipolar disorder. Nat. Genet. 51, 793-803 (2019).

34. Schizophrenia Working Group of the Psychiatric Genomics Consortium Biological insights from 108 schizophrenia-associated genetic loci. Nature $\mathbf{5 1 1}$ 421-427 (2014).

35. Howard, D. M. et al. Genome-wide meta-analysis of depression identifies 102 independent variants and highlights the importance of the prefrontal brain regions. Nat. Neurosci. 22, 343-352 (2019).

36. Demontis, D. et al. Discovery of the first genome-wide significant risk loci for attention deficit/hyperactivity disorder. Nat. Genet. 51, 63-75 (2019).

37. Purves K. L. et al. A major role for common genetic variation in anxiety disorders. Mol. Psychiatry. https://doi.org/10.1038/s41380-019-0559-1 (2019).

38. International Obsessive Compulsive Disorder Foundation Genetics Collaborative (IOCDF-GC) and OCD Collaborative Genetics Association Studies (OCGAS). Revealing the complex genetic architecture of obsessive-compulsive disorder using meta-analysis. Mol. Psychiatry 23, 1181-1188 (2018).

39. Watson, H. J. et al. Genome-wide association study identifies eight risk loci and implicates metabo-psychiatric origins for anorexia nervosa. Nat. Genet. 51, 1207-1214 (2019).

40. Kranzler, H. R. et al. Genome-wide association study of alcohol consumption and use disorder in 274,424 individuals from multiple populations. Nat. Commun. 10, 1-11. (2019).

41. Lane J. M. et al. Biological and clinical insights from genetics of insomnia symptoms. Nat. Genet. 51, 387-393 (2019).

42. Lee, J. J. et al. Gene discovery and polygenic prediction from a genome-wide association study of educational attainment in 1.1 million individuals. Nat Genet. 50, 1112-1121 (2018).

43. Strawbridge R. J. et al. Genome-wide analysis of self-reported risk-taking behaviour and cross-disorder genetic correlations in the UK Biobank cohort. Transl. Psychiatry 8, 39 (2018).

44. Okbay, A. et al. Genetic variants associated with subjective well-being depressive symptoms, and neuroticism identified through genome-wide analyses. Nat. Genet. 48, 624-633 (2016).

45. Nagel, M. et al. Meta-analysis of genome-wide association studies for neuroticism in 449,484 individuals identifies novel genetic loci and pathways. Nat. Genet. 50, 920-927 (2018).

46. Ward, J. et al. Novel genome-wide associations for anhedonia, genetic correlation with psychiatric disorders, and polygenic association with brain structure. Transl. Psychiatry 9, 1-9 (2019).

47. UK Biobank-Neale lab. http://www.nealelab.is/uk-biobank. Accessed 24 Feb 2020 . 
48. Bulik-Sullivan, B. et al. LD score regression distinguishes confounding from polygenicity in genome-wide association studies. Nat. Genet. 47, 291-295 (2015).

49. Dudbridge F., Cole C. B., Palla L. avengeme: Analysis 1.0., of polygenic scoring methods (R package version, 2019).

50. Ge, T., Chen, C. Y., Ni, Y., Feng, Y. C. A. \& Smoller, J. W. Polygenic prediction via Bayesian regression and continuous shrinkage priors. Nat. Commun. 10, 1776 (2019).

51. Choi S. W. \& O'Reilly P. F. PRSice-2: Polygenic Risk Score software for biobankscale data. Gigascience. 8, giz082 (2019).

52. Chang, C. C. et al. Second-generation PLINK: rising to the challenge of larger and richer datasets. Gigascience 4, 7 (2015).

53. Kupka, R. W., Luckenbaugh, D. A., Post, R. M., Leverich, G. S. \& Nolen, W. A. Rapid and non-rapid cycling bipolar disorder: a meta-analysis of clinical studies. J. Clin. Psychiatry 64, 1483-1494 (2003).

54. Mullins, N. et al. GWAS of suicide attempt in psychiatric disorders and association with major depression polygenic risk scores. Am. J. Psychiatry $\mathbf{1 7 6}$ 651-660 (2019).

55. Black, D. W. \& Nasrallah, A. Hallucinations and delusions in 1,715 patients with unipolar and bipolar affective disorders. Psychopathology 22, 28-34 (1989).

56. Strassnig, M. et al. Twenty-year progression of body mass index in a county-wide cohort of people with schizophrenia and bipolar disorder identified at their first episode of psychosis. Bipolar Disord. 19, 336-343 (2017).
57. Caravaggio, F. et al. Exploring the relationship between body mass index and positive symptom severity in persons at clinical high risk for psychosis. J. Nerv. Ment. Dis. 205, 893-895 (2017).

58. Ikeda, M. et al. Re-evaluating classical body type theories: Genetic correlation between psychiatric disorders and body mass index. Psychol. Med. 48, 1745-1748 (2018).

59. Kalman J. L. et al. Investigating polygenic burden in age at disease onset in bipolar disorder: findings from an international multicentric study. Bipolar Disord. 21, 68-75 (2019)

60. Brent, D. A. et al. Familial transmission of mood disorders: convergence and divergence with transmission of suicidal behavior. J. Am. Acad. Child Adolesc Psychiatry 43, 1259-1266 (2004).

61. Aedo, A. et al. Clinical characterization of rapid cycling bipolar disorder: Association with attention deficit hyperactivity disorder. J. Affect Disord. 240, 187-192 (2018)

62. Kupka, R. W. et al. Comparison of rapid-cycling and non-rapid-cycling bipolar disorder based on prospective mood ratings in 539 outpatients. Am. J. Psychiatry 162, 1273-1280 (2005).

63. Oquendo, M. A. et al. Suicidal behavior in bipolar mood disorder: clinical characteristics of attempters and nonattempters. J. Affect Disord. 59, 107-117 (2000).

64. Valtonen, H. M. et al. Prospective study of risk factors for attempted suicide among patients with bipolar disorder. Bipolar Disord. 8, 576-585 (2006).

65. Ducasse, D. et al. Anhedonia is associated with suicidal ideation independently of depression: a meta-analysis. Depress. Anxiety 35, 382-392 (2018). 